Maciej Jędrusik

\title{
QUELQUES MODÈLES DE COLONISATION TOURISTIQUE CONTEMPORAINE
}

La colonisation touristique (l'utilisation de ce terme nous semble justifiée lorsqu'il s'agit de la pénétration des touristes ou des investissements touristiques dans les nouvelles contrées) entraîne non seulement des changements économiques, mais des changements sociaux et culturels profonds, difficiles à mesurer. Elle s'effectue, le plus souvent, sous l'influence de gens étrangers à la région colonisée. C'est eux qui créent la demande et investissent dans les services touristiques. Comme dans le cas de la colonisation classique, les différences dans le déroulement de la colonisation touristique peuvent être notables, en fonction, entre autres, de la participation de la population locale dans la mise en valeur des nouveaux terrains, d'où quelques modèles de colonisation touristique contemporaine.

Dans le premier modèle la mise en valeur touristique consiste à former des enclaves entièrement séparées de leur entourage. Les enclaves touristiques sont créées par le capital étranger, les touristes ne les quittent qu'accidentellement, les contacts avec la population locale restent rares et limités, les bénéfices sont transférés en dehors de l'espace colonisé, le développement touristique n'a pratiquement aucune influence sur le développement de la région. En principe, deux espaces commencent à exister: l'espace touristique et celui de la population locale. Entre eux, il n'y a presque pas de contact.

Dans d'autres modèles, la population locale prend part dans le processus de colonisation et participe aux bénéfices. Le degré de son engagement peut être différent. Dans, le deuxième modèle la colonisation touristique vient de l'extérieur. C'est le capital extérieur qui crée les premiers investissements touristiques et organise le mouvement touristique. Avec l'arrivée des touristes, les marchandises et les services locaux sont de plus en plus demandés, l'engagement de la population augmente. L'économie évolue aussi bien dans les terrains touchés par le capital étranger que dans les terrains limitrophes. Une partie des bénéfices est transférée à l'étranger, mais une partie, surtout au début, contribue au développement et sert à la restructuration de l'économie de la région colonisée.

Dans le troisième modèle, la colonisation touristique vient de l'intérieur. C'est grâce à la population locale que les premiers investissements touristiques 
apparaissent. L'offre des services touristiques crée la demande. Elle attire le capital extérieur qui investit dans l'infrastructure touristique.

Ces modèles évoluent, mais il est difficile de déterminer la direction de leurs changements. Notons que toutes ces activités conduisent à des déséquilibres: au développement des régions mises en valeur par le mouvement touristique, et à la stagnation des régions limitrophes. Mais n'était-ce pas un aspect inhérent à chaque colonisation?

Chacun de ces modèles entraîne une autre fonction dans la région colonisée, un autre niveau de vie des habitants et un autre degré de leur participation aux bénéfices obtenus grâce au développement touristique. Du point de vue des conséquences de ce développement, il est donc possible d'appeler ces modèles: isolement, symbiose et parasitisme.

Les îles tropicales nous fournissent de très beaux exemples de ces trois modèles de colonisation touristique. Le premier est caractéristique des Maldives, le second - des îles thaïlandaises, le troisième - en Polynésie française, surtout de l'archipel de Tuamotu.

\section{LES DEUX MONDES DES MALDIVES}

Les débuts du développement touristique dans les Maldives datent des années soixante et soixante-dix du $\mathrm{XX}^{\mathrm{e}} \mathrm{s}$. Avant, les contacts avec l'archipel étaient très limités. Il n'y avait aucune liaison aérienne, aucune liaison téléphonique. La première piste d'atterrissage, de $1400 \mathrm{~m}$ seulement, n'a été construite qu'en 1968, les premiers villages de vacances - en 1972. Les contacts avec le monde extérieur se sont développés grâce à l'extension de l'aéroport Hulule près de Male et des ports de Hulule et de Male. Les "îles-hôtels" se trouvaient au début à proximité de l'aéroport, mais, petit à petit, la zone hôtelière s'est élargie, occupant progressivement les autres îles, en commençant par celles des atolls de Male. En 1981, la piste de l'aéroport a été allongée jusqu'à $2840 \mathrm{~m}$, ce qui a permis l'arrivée de gros avions, venant directement d'Europe. Les dhoni, bateaux à moteur, lents et petits, assurant le transport entre les différentes îles, ont été remplacés par des bateaux rapides et des hydroglisseurs, des hélicoptères et des hydravions. Tout ceci a facilité l'occupation des atolls de plus en plus éloignés de Male et le projet de la mise en valeur de Gan - la plus grande île de l'archipel, située dans le Sud. Grâce à l'aéroport qui y sera construit, les Maldives, ce véritable paradis touristique, pourront accueillir près de 800000 touristes, plus que n'importe quel autre archipel de l'Océan Indien.

Le développement de l'infrastructure et l'augmentation du mouvement touristique dans les Maldives ont donc été très dynamiques. La progression de la zone de la mise en valeur touristique, depuis les atolls de Male vers les îles de plus en plus éloignées, porte les signes caractéristiques de la colonisation, qui, jadis, consistait à conquérir des espaces lointains. Dans le cas des Maldives, cette conquête est particulière car il s'agit d'îles inhabitées. Leur milieu naturel s'est presque totalement transformé au cours des étapes 
suivantes: 1) construction d'un embarcadère et d'un accès aux installations; 2) agrandissement du territoire de l'île (33 seulement des 1200 îles faisant partie de l'archipel ont la superficie dépassant $1 \mathrm{~km}^{2}$; île la plus grande, Gan, n'a que $5,2 \mathrm{~km}^{2}$; les îles situées à proximité de Male, particulièrement petites, ont été agrandies à l'aide des matériaux obtenus pendant la construction et l'approfondissement des ports) ; 3) création de la plage (elle était nécessaire dans la moitié env. des 87 “îles-hôtels"); 4) défense de l'île, de l'embarcadère et de la plage contre l'érosion.

Ensuite, la végétation a été transformée. Les cocotiers ont été décimés, car les noix, tombant des arbres, représentaient un danger pour les touristes. Une bonne partie des broussailles composées des pandanus et des arbres à pain a été remplacée par des arbustes et des arbres décoratifs. Sur certaines îles on a créé des jardins potagers.

La préparation d'une île pour y établir un village de vacances est toujours coûteuse. Les coûts augmentent au fur et à mesure que les établissements deviennent plus luxueux. En 1973, il fallait dépenser env. 900 dollars pour créér un lit de village de vacances, en $1985-21000$ dollars, au début des années quatre-vingt-dix - 33000 dollars. Le dessalement de l'eau de mer favorise la construction des piscines ou l'utilisation des jacuzzis, l'eau chaude courante et la climatisation sont partout disponibles (par contre, curieusement, la télévision par satellite est rare).

L'amélioration de la qualité constitue, après l'expansion territoriale, le deuxième trait caractéristique de la colonisation touristique des Maldives. Les différences entre l'équipement des "îles-hôtels" et les conditions de vie de la population locale sont énormes et c'est peut-être pour cela que, depuis 1980, les autorités mènent une politique de stricte séparation des touristes; cette politique est favorisée par le fait que les îles mises en valeur appartiennent à l'État. Ainsi, par exemple, les Maldiviennes ne peuvent pas séjourner dans les centres touristiques après $18.00 \mathrm{~h}$, la même interdiction concerne les étrangers dans les îles non touristiques. Les immigrants de l'Inde, du Bangladesh ou du Sri Lanka constituent l'essentiel du personnel employé dans les "îles-hôtels", où le temps légal diffère d'ailleurs du temps légal des Maldives (pour que les touristes puissent diner au coucher du soleil et dormir plus longtemps dans leurs chambres) et où la vente et la distribution de l'alcool sont admises.

Les raisons de l'existence de ce modèle, d'une telle séparation de deux mondes, résident non seulement dans la volonté de préserver la culture musulmane locale de l'invasion étrangère. Il s'agit aussi de l'attitude des élites maldiviennes. Elles investissent, avec les étrangers, dans le tourisme, et ne veulent pas partager les bénéfices avec la population locale (Gay, 2001). En réalité donc, malgré le développement spectaculaire du tourisme maldivien, ceux qui n'y sont pas directement impliqués n'en profitent guère. Pour résumer, ce modèle d'isolement se caractérise par:

- les investissements notables du capital étranger,

- l'infrastructure dominée par le capital extérieur et les immigrants, 
- une faible participation de la population locale dans les services touristiques,

- le transfert des bénéfices à l'étranger,

- les changements faibles ou inexistants dans la structure de l'économie et dans l'emploi de la population locale,

- les changements faibles ou inexistants dans le niveau de vie de cette population.

\section{LA SYMBIOSE THAÏLANDAISE DES GRANDS ET DES PETITS}

Parmi les nombreuses îles situées au large de la Thaïlande, celles qui se trouvent dans la Mer des Andaman attirent surtout l'attention. C'est là que la colonisation touristique du Sud du pays a commencé au début des années soixante-dix, à partir de l'île de Phuket. Les terrains de camping bon marché étaient les premiers à s'installer sur les meilleures plages. Le Club Méditerranée est arrivé au début des années quatre-vingts. Ensuite Phuket a attiré les capitaux étrangers: en 1994, les établissements touristiques, au nombre de 264, disposaient déjà de 17000 lits. Enfin, les îles voisines, plus petites, ont commencé à intéresser les touristes; parmi eux il y avait aussi des Thaïlandais. Certains ont installé des salons de massage et des restaurants. Les étrangers (Australiens, Allemands, Suisses, Italiens) sont aussi présents.

Ces changements économiques ont eu des conséquences sur le niveau de vie de la population locale. Chez certains, la situation n'a pourtant pas beaucoup changé. Les contrastes sont d'autant plus visibles que les terrains habités occupent de très faibles superficies. Ton Sai, par exemple, abrite $90 \%$ de la population et $80 \%$ des touristes sur à peine $1 \mathrm{~km}^{2}$. Dans les hôtels et les campings l'adduction d'eau est assurée, mais les habitants locaux utilisent toujours des puits de mauvaise qualité. Donc, comme dans les Maldives, la population locale ne profite pas beaucoup des bénéfices dus au développement du tourisme. Mais c'est la seule ressemblance, car la structure de l'emploi a été profondément changée. Les hommes plus âgés sont toujours pêcheurs, mais certains se spécialisent dans les excursions et dans la pêche organisée pour les touristes. Les jeunes s'occupent de différents services, gèrent les hôtels et les campings, font le commerce.

Phuket, devenue centre de mouvement touristique, a stimulé le développement des îles voisines: Phi Phi Don et Lanta Yai. Cette colonisation touristique s'est caractérisé par:

- des investissements importants du capital étranger;

- l'emploi de la population locale dans les services touristiques;

- la création d'une bonne partie de l'infrastructure (surtout concernant l'hébergement meilleur marché) par la population locale;

- les changements dans la structure de l'économie et dans l'emploi de la population locale (l'abandon de l'exploitation des cassitérites à Phuket, diminution du rôle de l'agriculture, élevage des crevettes à Lanta Yai); 
- l'amélioration de l'infrastructure technique (électrification et construction des routes à Lanta Yai);

- l'amélioration de la qualité des établissements et des services touristiques,

- l'amélioration du niveau de vie des habitants (Phuket est une des provinces les plus riches du pays et le mieux équipée dans le domaine de l'infrastructure sociale).

Contrairement au modèle d'isolement observé dans les Maldives, le modèle de la colonisation touristique des îles thaïlandaises de la Mer des Andaman peut être considéré comme celui de symbiose. Une partie des bénéfices est réinvestie sur place, la population locale profite du développement du tourisme. Notons aussi que l'ingérence dans le milieu naturel est beaucoup moins importante que dans les Maldives. Ces différences sont donc notables bien que, dans les deux cas, la colonisation touristique soit due à une impulsion venant de l'extérieur.

\section{TUAMOTU — LA RECHERCHE D'UN MEILLEUR AVENIR}

La coexistence, comme celle qui vient d'être décrite en Thaïlande, peut se transformer en parasitisme. Dans le cas des activités touristiques il arrive que, dans une région desservie par des petits établissements, apparaît un grand hôtel de luxe qui absorbe progressivement la clientèle et conduit au déclin du secteur "traditionnel". L'impulsion vient de l'intérieur.

Il est possible d'observer une telle situation à Tuamotu, en Polynésie française, surtout au Nord et à l'Ouest de l'archipel, où se concentre le mouvement touristique. L'infrastructure, plutôt modeste, s'est développée surtout dans les atolls de Rangiroa, Tikehau, Matai et Fakarava. En 2000, il n'y avait que 367 maisonnettes ou chambres d'hôtel, dont presque la moitié à Rangiroa (La Polynésie française, 2001); parmi ces unités, $60 \%$ appartenaient à la catégorie des pensions de famille.

Cette structure est compréhensible. Le développement touristique des Tuamotu s'est fait à la demande de la population locale, soutenue par les autorités de la Polynésie française. Dans les années soixante-dix, l'archipel a vécu une crise économique profonde. La production de coprah, l'exploitation des phosphates et la production de la nacre ont pratiquement disparu; l'exode vers les Îles de la Société s'est accru. Après la fabrication des perles de culture, on a donc misé sur le tourisme. Son développement a commencé avec la construction de l'aéroport à Rangiroa, en 1965. Les touristes se sont installés dans les petites pensions créées par la population locale. En 2000, il y avait 20 pensions de ce genre à Rangiroa (sur 46 dans tout l'archipel). Le premier hôtel de classe supérieure n'a été construit que dans les années quatre-vingtdix, et à Tikehau et Fakarava - seulement en 2001. Ces hôtels ont transformé l'espace touristique de Tuamotu et absorbé une partie du mouvement touristique, au détriment des propriétaires des pensions de famille. Une autre partie des bénéfices, à part l'hébergement, a été saisie par la restauration. 
Ces constructions n'ont été profitables ni pour les activités touristiques, ni pour l'archipel dans son ensemble, car les bénéfices obtenus ne semblent pas destinés à améliorer l'infrastructure technique. La situation donne des signes de parasitisme. Les étapes de ce modèle de colonisation touristique ont donc été les suivantes: aiguë;

- création des petites pensions de famille, après une crise économique

- augmentation du mouvement touristique; locale;

- changements des structures économiques et de l'emploi de la population

- amélioration du niveau de vie de cette population;

- construction d'un grand hôtel;

- perte d'une partie de la clientèle des pensions de familles;

- stagnation ou détérioration du niveau de vie de la population locale.

\section{LA COLONISATION TOURISTIQUE - ISOLEMENT, SYMBIOSE, PARASITISME}

Il est possible, d'après ces exemples de colonisation touristique, de déterminer les modèles d'isolement (les Maldives), de symbiose (la Thä̈lande) et de parasitisme (les Tuamotu). Bien que ces appellations aient un caractère valorisant, il ne serait pas opportun de juger lequel de ces modèles est le plus avantageux. Beaucoup dépend du point de vue du bénéficiaire. Le modèle qui ne donne rien à l'espace environnant peut être excellent pour les propriétaires des établissements touristiques, et pour les touristes. Un modèle apparemment neutre, comme celui d'isolement, peut être mal jugé par la population locale, car n'apportant pas de bénéfices. Les appréciations sont d'autant plus difficiles que les situations changent rapidement.

Les trois modèles de colonisation touristique n'illustrent certainement pas toutes les situations. Ils ne sont pas, soulignons-le, caractéristiques uniquement des îles tropicales. Ne serait-ce qu'en Pologne, il ne serait pas difficile de trouver des exemples d'isolement, de symbiose et de parasitisme.

Remarquons enfin la ressemblance entre les processus de la mise en valeur touristique de diverses régions et les processus de la conquête coloniale. Cette conquête s'est terminée par la décolonisation. Comment se déroulera la décolonisation dans le cas de l'espace touristique?

\section{BIBLIOGRAPHIE}

G a y J.-Ch., 2001, Lîle-hôtel, symbole du tourisme maldivien, Les Cahiers d'Outre-Mer, 213, $26-52$.

La Polynésie française en 2000, 2001, Paris. 